\title{
L'organisation de la radioprotection du personnel des entreprises extérieures au Centre de Protection Nucléaire de Gravelines*
}

\author{
R. CAPEL** \\ (Manuscrit reçu le 3 mai 1985)
}

RESUME

Cet article a pour objectif de présenter comment la radioprotection des travailleurs des entreprises extérieures est organisée au niveau de l'exploitation d'une centrale d'Electricité de France.

\section{ABSTRACT}

The purpose of this paper is to present how radiation protection of contractors' workers is organised during operation of an EdF power plant.

Après une brève présentation qui situe le problème dans le cadre général des activités de production d'électricité, l'auteur dresse le bilan de quatre années d'expérience en termes d'accueil des entreprises, de formation et d'habilitation du personnel, de suivi des chantiers, de contrôle de la dosimétrie, de vécu des problèmes, enfin de retour d'expérience, c'est-à-dire recherche permanente de l'amélioration des pratiques et de la réduction des doses.

\section{INTRODUCTION}

Le centre de production nucléaire (CPN) de Gravelines est constitué de 6 tranches de $900 \mathrm{MW}$, réacteur à eau pressurisée (REP), type Westinghouse.

Situé à une trentaine de kilomètres de la frontière belge, sur la mer du Nord, à mi-chemin entre Dunkerque et Calais, c'est actuellement, avec 5 tranches en exploitation, le plus gros centre européen de production d'électricité d'origine nucléaire.

Lorsque la $6^{e}$ tranche entrera en service cet été (1985), ce sera le plus gros centre mondial. Trois tranches fonctionnent depuis 1980, une depuis 1981 et la $5^{\text {e }}$ depuis l'année dernière. A ce jour, 10 révisions de tranches

* Communication présentée au colloque organisé à Bruxelles, du 20 au 22 mars 1985 . par les Sociétés belge et française de radioprotection, sur la "Radioprotection du personnel des entreprises extérieures intervenant dans les installations nucléaires".

$\star \star$ EdF, Service de la Production Thermique, Centre de Production Nucléaire de Gravelines, BP 77, 59820 Gravelines. 
ont été réalisées. Environ 1400 agents assurent l'exploitation de cet ensemble se répartissant ainsi : $30 \%$ pour le personnel de conduite, $60 \%$ pour le personnel de maintenance, et enfin $10 \%$ pour le personnel administratif, comptable, financier. En termes de qualification, la répartition est la suivante : $10 \%$ de cadres, $60 \%$ d'agents de maîtrise, $30 \%$ d'agents d'exécution. II s'agit, par ailleurs, d'une population jeune, puisque l'âge moyen est de 31 ans.

Si ce personnel est suffisant pour exploiter normalement les tranches, il faut, bien sûr, faire appel aux entreprises pour mener à bien les révisions annuelles (entretien des matériels, inspection des circuits, renouvellement du combustible), dont la durée moyenne pour un arrêt normal est de 5 à 7 semaines par tranche.

En termes de production, le centre de production de Gravelines a fourni l'année dernière au réseau 26 milliards de $\mathrm{kWh}$, c'est-à-dire de quoi alimenter toute la région Nord/Pas-de-Calais : 20 milliards environ, et exporter le reste sur le réseau national.

En 1984, ceci représente :

- $8,4 \%$ de la production française d'électricité : 310 TWh ;

- $12,1 \%$ de la production thermique EdF : $215 \mathrm{TWh}$;

- $14,5 \%$ de la production nucléaire EdF : $180 \mathrm{TWh}$.

Exprimée en énergie de remplacement, cette production a permis l'économie de près de $6000000 \mathrm{t}$ de pétrole ou encore $9000000 \mathrm{t}$ de charbon. A titre de comparaison, les Charbonnages de France ont produit l'année dernière $18000000 \mathrm{t}$ de charbon. Gravelines fonctionnant au charbon aurait consommé près de la moitié de la production nationale, ou plus de 3 fois la production du bassin houiller du Nord et du Pas-de-Calais.

\section{PLACE DE LA RADIOPROTECTION DANS L'EXPLOITATION DE LA CENTRALE}

On entend souvent dire que la radioprotection est une spécialité. Or, en termes de sécurité, ne parler que de radioprotection et n'en parler qu'en termes de spécialiste, ou autrement dit, dans nos installations, confier cette activité à des experts, c'est oublier deux choses : la radioprotection n'est qu'un des aspects de la sécurité en général, la radioprotection est l'affaire de tous. C'est dans cet esprit que sont organisées les sections "Sécurité radioprotection (SRP)" : une douzaine d'agents par paire de tranches.

L'objectif n'est pas de réveiller ici de vieilles querelles. La radioprotection est à la fois une discipline scientifique et une activité professionnelle. II faut reconnaître que les radioprotectionnistes ont fait faire, ces dernières décennies, des progrès spectaculaires dans ce domaine. La qualité de leurs recherches a sans aucun doute conduit à rendre l'industrie nucléaire, qui est potentiellement dangereuse, peut-être la plus sûre qui soit.

II n'est surtout pas question de dire : à quoi bon privilégier une discipline apparemment sans objet, puisque les incidents radioactifs sont 
exceptionnels, et les risques classiques, par contre, bien plus préoccupants? Le fait est là.

Le propos veut simplement traduire le sentiment que les experts ont réussi à mettre au point un ensemble de dispositions qu'il revient aux responsables de faire passer dans les mœurs, de faire accepter par les agents et les intervenants comme pratique courante dans les règles de sécurité. C'est la mission essentielle qui incombe à EdF en matière de radioprotection. Dans ces conditions, on comprend aisément que la doctrine EdF vise à ce que tous les agents soient formés et se sentent personnellement concernés. EdF insiste auprès des entreprises pour qu'elles acceptent cette façon de voir.

Les sections SRP ne sont pas des services spécialisés, chargés de tout savoir, de tout faire et de surveiller en permanence tout un chacun. Leur rôle peut se résumer ainsi : assistance, conseil, contrôle, formation, animation.

Il faut bien reconnaître que cette organisation donne satisfaction.

Le CPN de Gravelines n'a enregistré en quatre ans aucun accident d'origine radioactive. II faut toujours être prudent quand on annonce de tels résultats, mais pas au point, quand même, de ne pas les signaler.

\section{BILAN DE QUATRE ANNEES D'EXPLOITATION (10 REVISIONS DE TRANCHES)}

Ces quatre années d'exploitation se sont traduites par 10 révisions de tranches : 4 révisions de type décennal et 6 révisions de type normal, mais avec des chantiers "modifications" importants. Au bilan, ces révisions portent globalement sur 500000 heures.agents d'entreprises intervenant en zone contrôlée.

Une soixantaine d'entreprises interviennent en révision normale : 30 nationales (ou échelons régionaux d'entreprises nationales) et 30 locales ; elles comptabilisent $35000 \mathrm{~h}$ de travail en zone contrôlée, soit encore 200 personnes en moyenne par jour avec des pointes de 300.

\subsection{L'accueil des entreprises}

\section{- Echange d'informations préalables}

La réglementation française établit clairement la distinction entre la responsabilité de l'employeur (entreprise) et celle du chef d'établissement (EdF) :

- l'employeur est responsable de son personnel (classement DATR, suivi médical, formation, dosimétrie) ;

- EdF est responsable de l'organisation générale et de la pratique de la radioprotection dans l'établissement, c'est-à-dire qu'il assure la vérification de la qualification des intervenants, l'information sur les risques particuliers, le rappel des consignes locales, la fourniture de moyens de protection collective et individuelle, et la coordination des interventions.

Les prestations demandées par EdF font l'objet d'une commande détaillée. 
Préalablement à la première ouverture de chantier, le responsable d'entreprise et le responsable d'EdF échangent des informations sur les risques encourus, conviennent des mesures à prendre pour les éviter, et procèdent à une inspection commune des lieux. Tout ceci se trouve consigné dans un procès-verbal signé des deux parties. II incombe alors au responsable d'entreprise d'informer comme il convient l'ensemble des agents qu'il affecte au chantier. Un dossier des prescriptions de sécurité et des consignes locales est remis au responsable d'entreprise.

\section{- Accueil proprement dit des agents}

Afin de faciliter le contrôle qui incombe à EdF, un carnet individuel de radioprotection édité par le GIIN (Groupement intersyndical de l'industrie nucléaire) est remis à tout agent d'entreprise. II précise l'identité de l'agent, sa formation, son suivi médical ; il contient des indications dosimétriques, à caractère officieux, mais bien utiles cependant pour fournir des recoupements intéressants.

Une cellule d'accueil prend en charge les agents à l'entrée du site. Elle règle les problèmes administratifs et coordonne les actions des différents services intéressés.

L'agent doit, ensuite, suivre un ensemble de démarches. Tout d'abord, une vérification du carnet individuel est effectuée par la SRP. Ensuite, un contrôle des connaissances est réalisé (1 $1^{\text {re }}$ intervention et tous les ans), et une information sur les consignes locales d'intervention et de sécurité est donnée. Un diaporama d'une vingtaine de minutes est présenté qui rappelle :

- les principes généraux de radioprotection (nature des rayonnements, mesures fixes et mobiles, dosimétrie automatique) ;

- les conditions d'accès en zone contrôlée (ZC) (simulation accès, bâtiment des auxiliaires nucléaires) ;

- la conduite à tenir en cas d'accident (alerte chantier, locale, générale, plan du site, points de regroupement, numéros de téléphone à connaître) ; une plaquette "format de poche" est remise à ce sujet ;

- les principes de la protection site : zone surveillée, zone protégée, zone renforcée.

Depuis quelques temps, un nouveau film est projeté sur l'anthropogammamétrie, "l'Extralucide", son intérêt, son utilisation.

Le service médical assure le passage à l'anthropogammamètre pour le contrôle de la contamination interne à l'entrée sur le site.

Enfin, un badge d'accès personnalisé est établi uniquement pour la zone d'intervention prévue.

L'esprit général de toutes ces démarches est d'obtenir et de vérifier que les agents d'entreprise sont bien préparés à s'insérer dans l'organisation générale $E d F$, de façon à réduire les pertes de temps sur le chantier, minimiser les risques potentiels et concourir ainsi à la réduction des doses.

\subsection{La formation et l'habilitation des agents}

L'entreprise est responsable de la formation et de l'habilitation de son personnel. 
Une semaine avant le début des travaux, elle fournit la liste de ses agents (chefs de travaux et exécutants), et elle nomme un responsable, chef de chantier, chargé des relations avec EdF.

Pour chaque intervention, les agents doivent posséder les habilitations nécessaires: professionnelles et radioprotection. Les titres d'habilitation doivent pouvoir être présentés à tout moment.

En ce qui concerne les entreprises nationales, ceci ne pose pas trop de problèmes. EdF a établi des dossiers pédagogiques et les entreprises s'en inspirent pour former leur personnel.

Pour les entreprises locales, en ce qui concerne le site de Gravelines, des discussions ont eu lieu dès 1978, c'est-à-dire deux ans avant le démarrage de la $1^{\text {re }}$ tranche et trois ans avant la première révision, entre EdF et la Chambre de Commerce et d'Industrie de Dunkerque, traitant au nom de toutes ces entreprises locales inexpérimentées alors en matière de radioprotection, mais désireuses d'intervenir dans les installations. Des contacts ont été pris avec des sociétés régionales de formation (APAVE, SIFOP (1) qui ont demandé à EdF de leur former des instructeurs pour former eux-mêmes, ensuite, les agents d'entreprises selon les modules EdF RP1 - RP2, c'est-à-dire formation en radioprotection des exécutants et des chefs de travaux.

Des formations spécifiques ont également été lancées, telles que : montage des sas, habillage, déshabillage du personnel, procédé de décontamination des matériels, nettoyage des surfaces, exploitation des matériels de mesures, etc.

Pour les interventions particulières comme, par exemple, les interventions dans les boîtes à eau des générateurs de vapeur, des exercices de répétition sur maquette ont été organisés. De la même façon, ont été programmées des sessions de recyclage. Un contact permanent s'est établi entre ces organismes de formation, les entreprises et EdF, qui permet de parfaire la finalité de toutes ces actions.

Depuis 1979 , un rythme soutenu des sessions a permis la formation d'un grand nombre d'agents : souvent, d'ailleurs, les mêmes qui, intervenant alors sur le site en construction, prenaient, de ce fait, connaissance des circuits et des matériels sur lesquels ils allaient être appelés plus tard à intervenir, mais cette fois-ci en zone contrôlée.

\subsection{Le suivi des chantiers}

Aucun travail ne peut commencer sans que le chef de travaux ne soit en possession d'un ordre d'exécution de travaux, auquel est joint le dossier technique d'intervention, et d'une attestation de consignation (AC) qu'il retire auprès du service d'exploitation.

A cette occasion, une concertation directe s'établit entre chef de quart, chef de consignation et chef de travaux. Des informations sont échangées sur le matériel consigné et les conditions de l'intervention. II y a vérification en local de la concordance des manœuvres de consignation réalisées avec les travaux envisagés. Le chef de travaux marque son accord en visant l'attestation de consignation.

(1) Service interconsulaire de formation et de perfectionnement. 
En ce qui concerne le classement des zones contrôlées, verte, jaune, orange ou rouge, celles-ci sont définies et délimitées par la SRP. Une cartographie des lieux est réalisée avant l'intervention, qui précise les débits d'exposition, les contaminations surfaciques, les points chauds.

L'ouverture du chantier intervient après ventilation des locaux, nettoyage des zones contaminées, réduction des points chauds. Le chef de travaux est averti des risques potentiels que peut induire son intervention et de ceux des chantiers voisins. Des balises de contrôle, aérosols en particulier, sont mises en place.

L'ouverture d'un chantier en zone contrôlée ne modifie pas le classement de celle-ci. Le chantier constitue une zone particulière bien délimitée dont les frontières sont nettes et dont l'accès ne peut se faire qu'avec l'accord et sous la responsabilité du chef de travaux. Cette zone temporaire disparaît avec le repli du chantier et après remise de l'attestation de consignation au service Exploitation. A ce propos, le chef de travaux est responsable de la remise en ordre du chantier après intervention et, en particulier, du tri et de l'évacuation des déchets radioactifs (vers un local approprié).

Les agents de la SRP assurent un suivi permanent des chantiers. Ils sont aisément repérables par leur tenue de couleur spéciale. Ils sont, en outre, équipés de récepteur radio "recherche de personnes" qui permet de les contacter à tout moment, où qu'ils soient. Les chantiers à risque sont visités journellement. Une cartographie générale des lieux est systématiquement reprise chaque semaine.

Des réunions spéciales se tiennent régulièrement, qui regroupent les chefs de chantiers et les responsables SRP qui font le point de l'évolution des chantiers, des problèmes rencontrés et des mesures prises ou à prendre, et des prévisions d'ouverture de nouveaux chantiers.

Cette organisation donne de bons résultats, même s'il faut souhaiter que les chefs de travaux soient mieux avertis encore de leur rôle et de leur responsabilité, et que soient améliorés, en particulier, le repli des chantiers et l'évacuation des déchets.

\section{LA DOSIMETRIE}

\subsection{L'organisation}

II faut rappeler qu'EdF est responsable de l'organisation générale de la radioprotection et, à ce titre, il assure la coordination des chantiers et l'échange d'information entre les divers intervenants. En particulier, il estime le coût approximatif dosimétrique de chaque intervention.

En ce qui concerne le suivi dosimétrique du personnel, il est à la charge de l'entreprise, mais pour lui faciliter la tâche, EdF effectue un suivi journalier automatique de cette dosimétrie.

Le système de recueil automatique des doses (fig. 1) permet de suivre, au jour le jour, la situation individuelle des agents, et donc de prévenir tout dépassement de dose, et de connaître le coût dosimétrique de chaque intervention. 


\section{Le système comprend :}

- un parc de dosimètres électroniques ;

- des appareils de saisie des informations aux entrées et sorties de zone (identification des agents, dose et code d'imputation de l'intervention) ;

- des "concentrateurs" locaux d'informations, reliés à un centralisateur de site, qui permet le traitement des données sur ordinateur.

Grâce à ce dispositif, une restitution journalière des anomalies (dose et code) est possible ; un bilan hebdomadaire : agents, chantiers, entreprises, est dressé ; un bilan global révision est établi. La figure 1 schématise le fonctionnement du système.

Avant d'entrer en zone contrôlée, chaque intervenant prend un dosimètre dans le rack, chargeur de batteries. A l'entrée de zone, il introduit son dosimètre, ainsi que son badge personnalisé d'accès au site, dans un lecteur d'entrée qui autorise le passage après avoir vérifié le bon fonctionnement du dosimètre et l'avoir commuté en position de comptage. En zone contrôlée, il peut, à tout instant et d'un simple coup d'œil, lire la dose reçue. II est averti de la présence d'un débit de dose anormalement élevé par un clignotant lumineux. A la sortie de zone contrôlée, il introduit son dosimètre et son badge dans le lecteur de sortie, frappe le code imputation de l'intervention.

Toutes les informations, entrées et sorties, sont transmises à un centralisateur de zone, muni d'une imprimante et à un centralisateur de site pour traitement sur ordinateur. Bien entendu, pour que ce système fonctionne correctement, il est indispensable que chaque intervenant en zone contrôlée soit muni de ces dosimètres électroniques et porte bien en sortie de zone contrôlée le code exact d'imputation de son activité (des efforts doivent encore être faits à ce sujet).

Pour l'aider, ce code est mentionné sur le dossier d'intervention, rappelé sur le chantier et inscrit en sortie de zone.

\subsection{Résultats}

Ils sont illustrés par les figures 2 à 7 et le tableau I.

\subsection{Les incidents (fig. 8 à 11)}

En cas de dépassement de la dose maximale admissible ou de contamination significative, c'est à l'employeur qu'il revient d'effectuer les démarches réglementaires auprès de l'Inspection du travail et du Service Central de Protection contre les Rayonnements Ionisants (SCPRI). II appartient, par contre, au chef de centrale d'assurer l'analyse de l'incident et de prendre toutes mesures utiles pour éviter son renouvellement.

Aucun incident notable n'est à signaler, depuis quatre ans, sur le site de Gravelines. Ce résultat favorable est dû pour une bonne part aux éléments développés dans le paragraphe suivant. Pour être quand même tout à fait exhaustif sur ce chapitre "incidents", il faut signaler que bon an, mal an, 150 cas légers de contamination corporelle sont relevés. En fait, un simple lavage ou un shampoing à l'infirmerie sont suffisants, pour 
éliminer la contamination observée. Ces incidents se produisent principalement lors du déshabillage. Des sessions d'entraînement sont fréquemment relancées à ce sujet. Ceci est néanmoins un point qu'il faut chercher en permanence à améliorer.

En cas de suspicion de contamination interne d'un agent, il est courant d'effectuer un certain nombre de prélèvements (frottis nasaux en particulier). Ces prélèvements n'ont qu'un rôle de dépistage. "Faut-il ou non envisager un passage à l'anthropogammamètre ?", seul véritable contrôle permettant de conclure ou non à une contamination interne (fig. 10 et 11).

Le matériel dont dispose le service médical a été défini par le SCPRI. Une unité comprend : 1 sonde "thorax" et 1 sonde "thyroïde", 1 analyseur multicanaux Canberra, 1 imprimante traçante, 1 calculateur. Le programme du calculateur est fourni par le SCPRI.

En sortie de zone, les agents subissent deux contrôles :

- une mesure $\gamma$ pour la contamination des tenues (portique $\mathrm{C} 1$ ) ;

- après déshabillage, une mesure $\beta$ pour la contamination corporelle (portique C2).

Si une contamination de la tête est relevée, la décontamination s'effectue à l'infirmerie ; elle peut être suivie ou non d'un passage à l'anthropogammamètre. S'il s'agit d'une contamination du corps, hors la tête, un nettoyage est effectué sur place et, s'il y a maintien de la contamination, l'agent est envoyé à l'infirmerie.

En accord avec le service général de médecine du travail, deux seuils de comptage à l'anthropogammamètre ont été définis (fig. 11) :

- le $1^{\text {er }}$ seuil (seuil d'enregistrement) correspond au $1 / 100^{e}$ de la QMA, au-dessous duquel on considère que l'agent n'est pas contaminé ;

- le $2^{e}$ seuil (seuil d'investigation) correspond au $1 / 30^{e}$ de la QMA, audessus duquel il convient d'effectuer des investigations complémentaires.

Pour les valeurs comprises entre ces deux seuils, il y a présomption d'une petite contamination interne, le fait est signalé par enregistrement sur le cahier d'infirmerie et l'agent reste bon pour le service. Le médecin du travail de l'agent est tenu informé du résultat du contrôle, avec les commentaires appropriés, par le médecin EdF.

Si le comptage est supérieur au $1 / 30^{\mathbf{e}}$ de la QMA, l'agent n'est plus autorisé à entrer en zone contrôlée. La conduite à tenir est différente selon qu'il s'agit : d'un contrôle effectué à l'arrivée sur le site, d'un contrôle effectué à l'occasion d'un chantier ou au départ de l'agent. Dans le premier cas, le médecin d'entreprise et le SCPRI sont informés de tous les résultats des examens pratiqués par le médecin EdF. Ils prennent la responsabilité des investigations complémentaires. Dans le deuxième cas, on considère que la contamination interne est consécutive aux travaux effectués. EdF en assure la responsabilité. II appartient alors aux services médicaux d'EdF d'appliquer les consignes établies pour le personnel EdF sur le plan de la thérapeutique préventive (administration d'iode stable, ou aérosol de DTPA) et sur le plan des investigations complémentaires, 
sauf si le médecin de l'entreprise ou le SCPRI, informés, revendiquent le soin de se livrer eux-mêmes à ces investigations.

Quelques petits problèmes sont survenus l'année dernière. Le SCPRI a modifié le programme du calculateur sans prévenir EdF, ni la médecine du travail. Le seuil d'enregistrement $\left(1 / 100^{\circ}\right.$ de la QMA) est devenu un seuil de "pré-investigation" et tout dépassement a entraîné l'envoi systématique au SCPRI d'agents qui présentaient une contamination interne légèrement supérieure à ce seuil (quelques $1 / 100^{\circ}$ de la QMA). L'impact psychologique a été fâcheux. Une meilleure collaboration de tous les intéressés aurait été souhaitable.

\section{LE RETOUR D'EXPERIENCE OU LA RECHERCHE PERMANENTE DE L'AMELIORATION DES MOYENS D'INTERVENTION ET DE REDUCTION DES DOSES}

Lorsque le réacteur est en service ou à l'arrêt, toute disposition d'exploitation est prise pour réduire la production de dépôts de corrosion radioactifs. La chimie de l'eau du circuit primaire a une répercussion directe sur l'activité des dépôts de corrosion que l'on trouve sur toutes les surfaces internes des circuits actifs. Ces dépôts sont à l'origine de la quasi-totalité des doses prises par le personnel. II est donc de la plus grande importance de chercher à en réduire l'activité.

En service, ceci est réalisé par un respect scrupuleux des spécifications techniques d'exploitation, notamment : surveillance des caractéristiques chimiques de l'eau primaire et $\mathrm{pH}$ en particulier, conditionnement à la lithine, suivi de l'activité du primaire, maintien de l'efficacité des filtres et des déminéralisateurs du système RCV, contrôle chimique et volumétrique.

A l'arrêt, la réduction de l'activité est obtenue, à la fois par un contrôle rigoureux de la phase "aération, oxygénation du primaire" qui donne lieu à un relâchement sous forme soluble d'une partie de l'activité (cobalt 58 principalement) déposée sur les structures, et par la garantie du bon fonctionnement et de l'efficacité des filtres et des déminéralisateurs du système PTR, du traitement et du refroidissement des eaux de piscine.

La réduction des doses passe également :

- par une prise en compte systématique de la radioprotection au stade de la préparation des interventions (établissement de fiches d'assistance, de fiches servitudes) ;

- par une généralisation de la pratique du "confinement dynamique des chantiers" : piégeage par aspiration de la contamination à la source même d'émission, mise en dépression des circuits ;

- par un entraînement des intervenants sur maquette (exemple : boîtes à eau des générateurs de vapeur, mise en place des tapes d'obturation) ;

- par une mise en œuvre de moyens techniques élaborés comme, par exemple, sas préfabriqués pour les générateurs de vapeur (GV), adaptés à la géographie des lieux avec confinement dynamique intégré, dispositif de rinçage permanent des parois piscine lors de la vidange, par ruissel- 
lement continu d'eau borée, traitement particulier des points chauds, (protections biologiques adaptées, filtrations mobiles, piégeage des matières en suspension).

L'emploi d'outillages spéciaux participe également à cette recherche de réduction des doses : machine de désserrage et serrage des goujons de cuve, dispositif de retrait des tampons, trous d'homme des GV, appareils de contrôle, bouchage, extraction des tubes GV, machine de débourrage des presse-étoupe des robinets, par eau sous forte pression, machine de nettoyage des plans de joints (brosse rotative avec captation des poussières), système de décontamination de pièces et de circuits aux ultrasons.

Pour en terminer avec ce chapitre, il faut noter que l'on assiste, depuis quelques temps, et un an en particulier, à une collaboration dans tous ces domaines, de plus en plus étroite, entre les entreprises et EdF, d'une part dans le cadre normal des relations réglementaires, par exemple les Comités spéciaux d'hygiène et de sécurité (CSHS), mais surtout, lors de contacts fréquents purement professionnels, dans l'intérêt réciproque des partenaires, soucieux de la gestion des hommes et de la gestion des moyens.

\section{CONCLUSION}

En guise de conclusion, il est bon de rappeler ce que disaient les responsables de la SENA, à l'issue de la première visite générale de la centrale de Chooz, en 1974 (des travaux d'envergure avaient été entrepris) :

"Nous avons rencontré beaucoup de problèmes, nous nous en sommes bien sortis. Mais de tels résultats n'ont pu être obtenus que moyennant l'affectation d'un personnel supplémentaire important aux tâches de radioprotection, soit pour les travaux d'intendance, soit pour l'encadrement du personnel d'entreprises.

"Cette nécessité se fait d'autant plus sentir, disaient-ils, que ce personnel d'entreprises n'est malheureusement pas encore au fait des conditions particulières qu'impose le travail en milieu radioactif. II faut souhaiter qu'une information suffisante soit donnée à l'avenir par chaque employeur au personnel qui devra intervenir dans les centrales en cours de mise en service".

Force est de reconnaître aujourd'hui, sans nier que de nouveaux progrès restent toujours nécessaires, que les entreprises ont en grande partie réalisé depuis dix ans cet effort d'information et de formation, alors souhaité. 
RADIOPROTECTION DU PERSONNEL DES ENTREPRISES EXTERIEURES A GRAVELINES

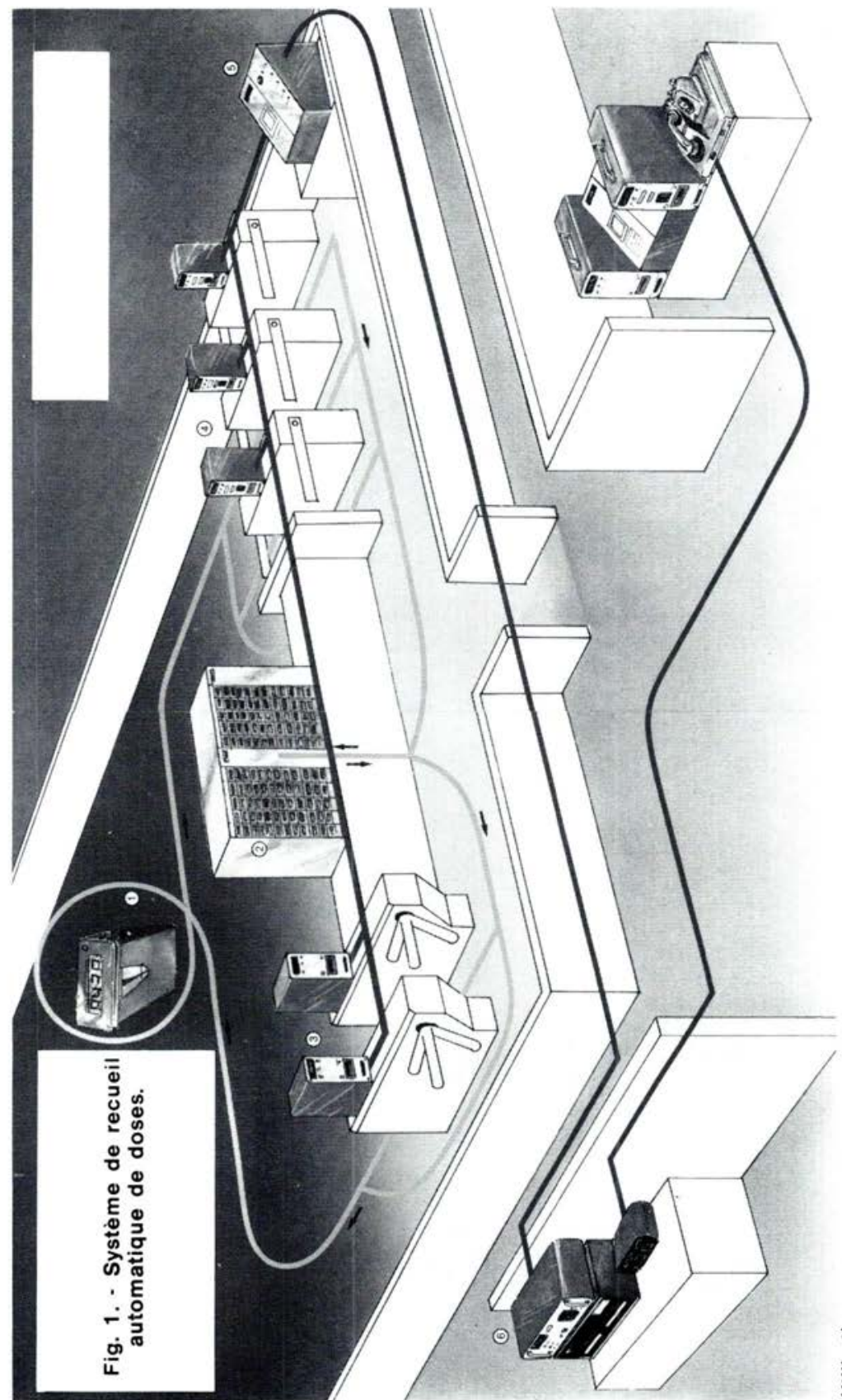

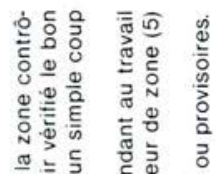

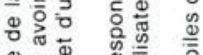

\&

क्ष

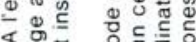

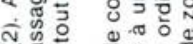

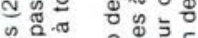

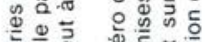

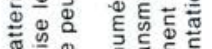

๑ั

का छ

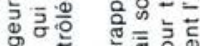

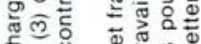

U.ष

记

๑听卫

क ป क है क्ष

तु

Ф

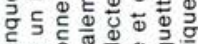

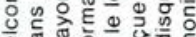

बiा

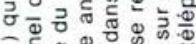

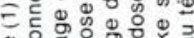

(⿻) क⿺辶万人

Ф है

心

음

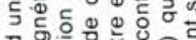

뮤융

ఏ

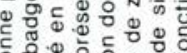

ธิษ

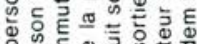

蛇。

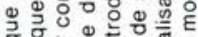

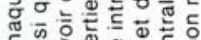

บ듀]

ఏळब के

은 $₫$

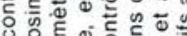

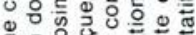

등ㅇㅁ웛

N

ब

$\Phi$ 잉

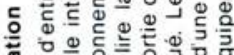

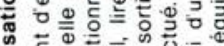

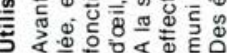

VOL. $21 \cdot \mathrm{N}^{\circ} 1$ 
R. CAPEL

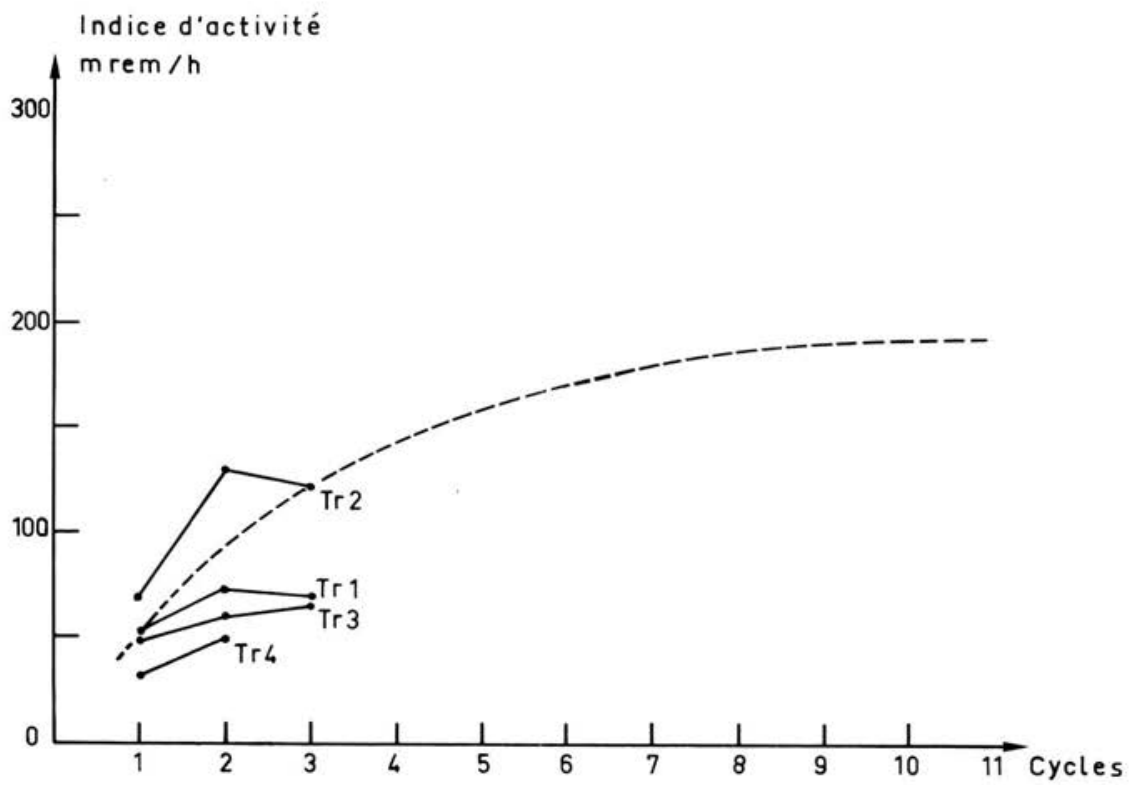

Fig. 2. - EdF-CPN Gravelines. Evolution de l'indice d'activité par tranche.

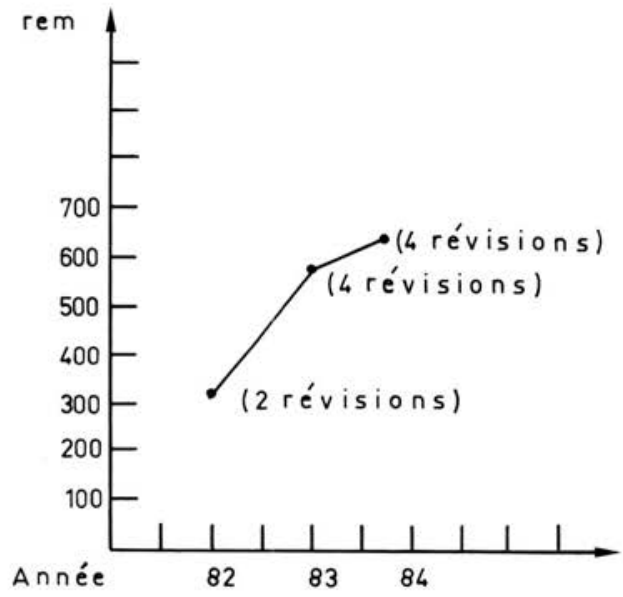

Fig. 4. - Evolution des doses annuelles globales "entreprises". 


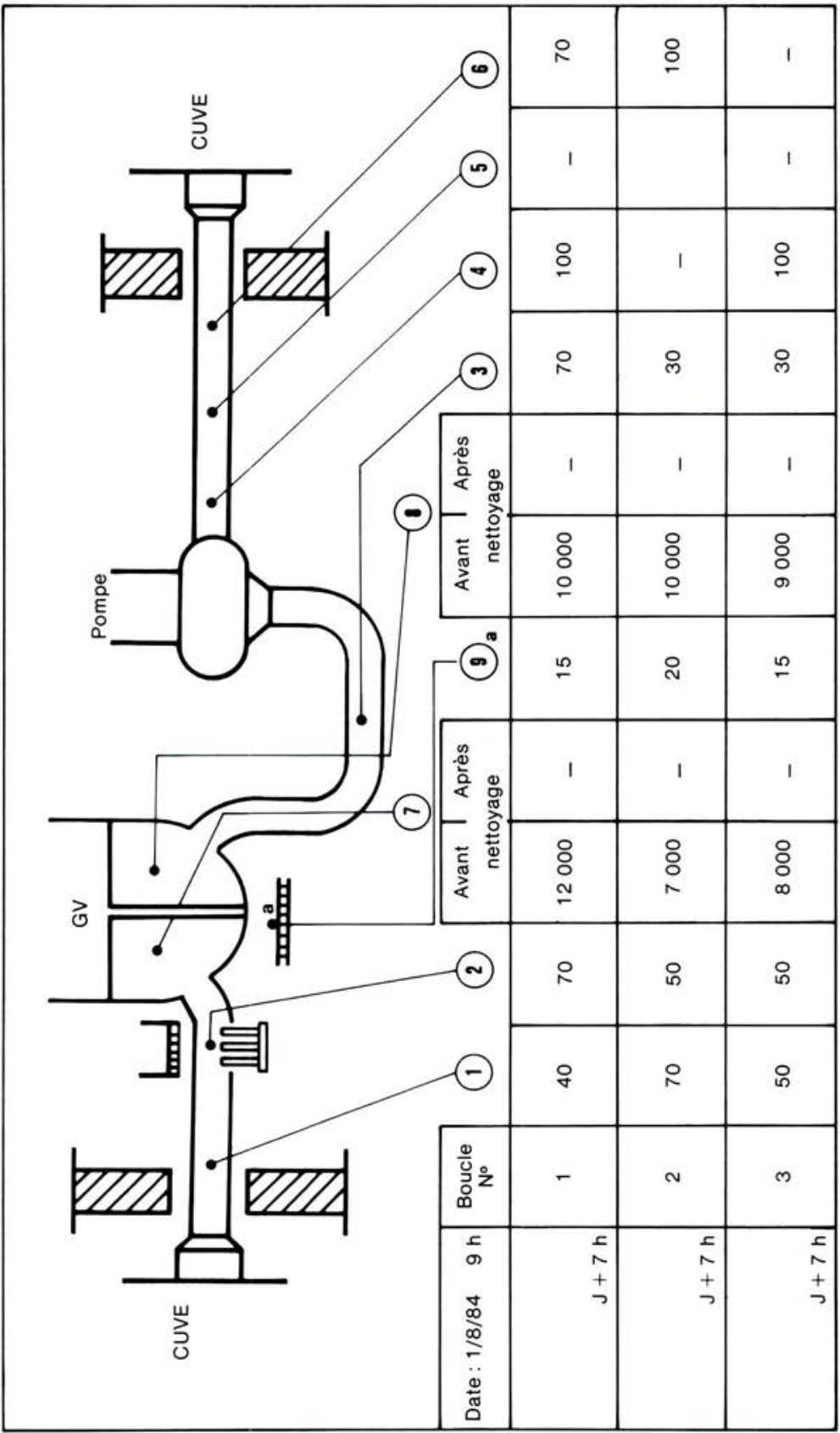

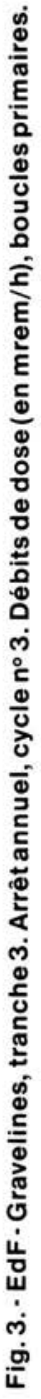


R. CAPEL

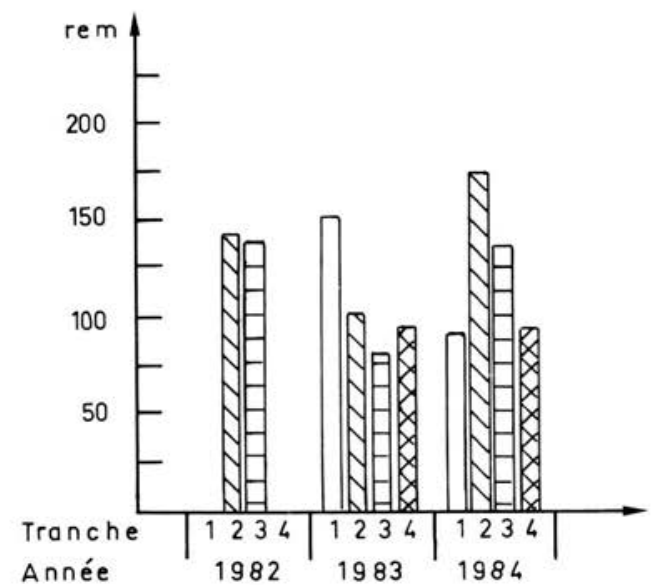

Fig. 5. - Doses " entreprises extérieures" pendant les arrêts annuels. Effectifs

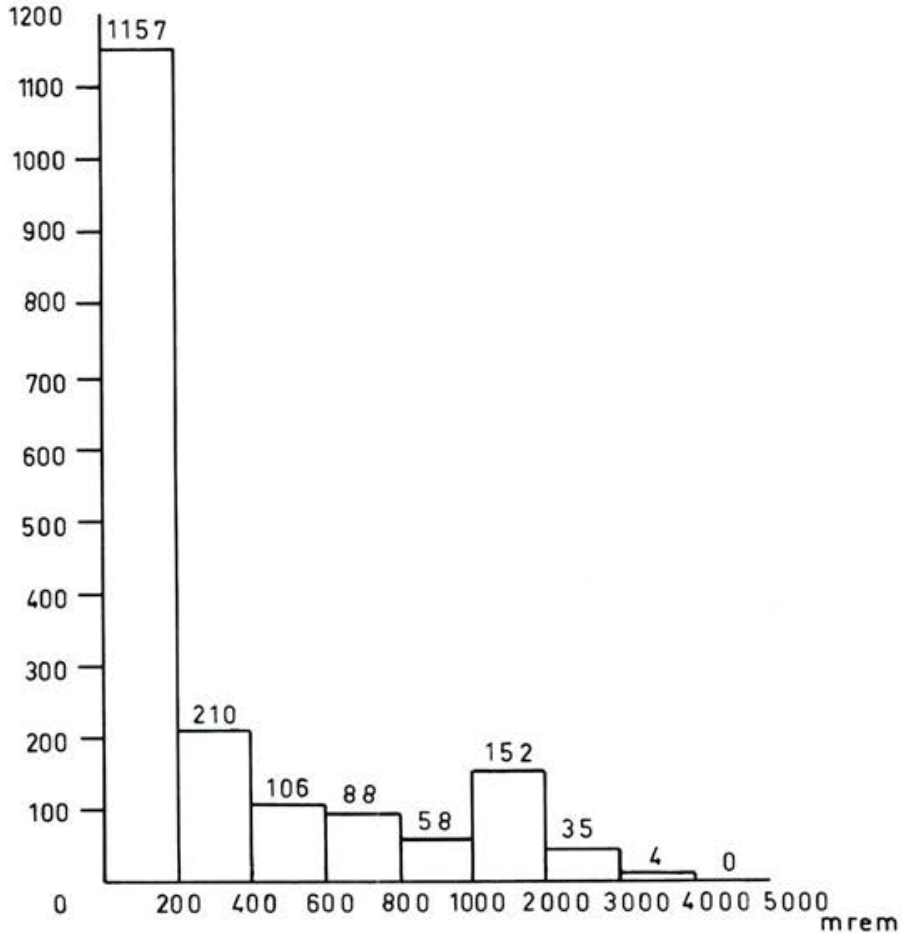

Fig. 6. - Répartition des doses/agent pour 1984 (toutes les entreprises). Nombre total d'agents d'entreprises : 1810 . Dose moyenne/agent d'entreprises : $380 \mathrm{mrem}$. Dose moyenne/agent EdF : 250 mrem. 
RADIOPROTECTION DU PERSONNEL DES ENTREPRISES EXTERIEURES A GRAVELINES

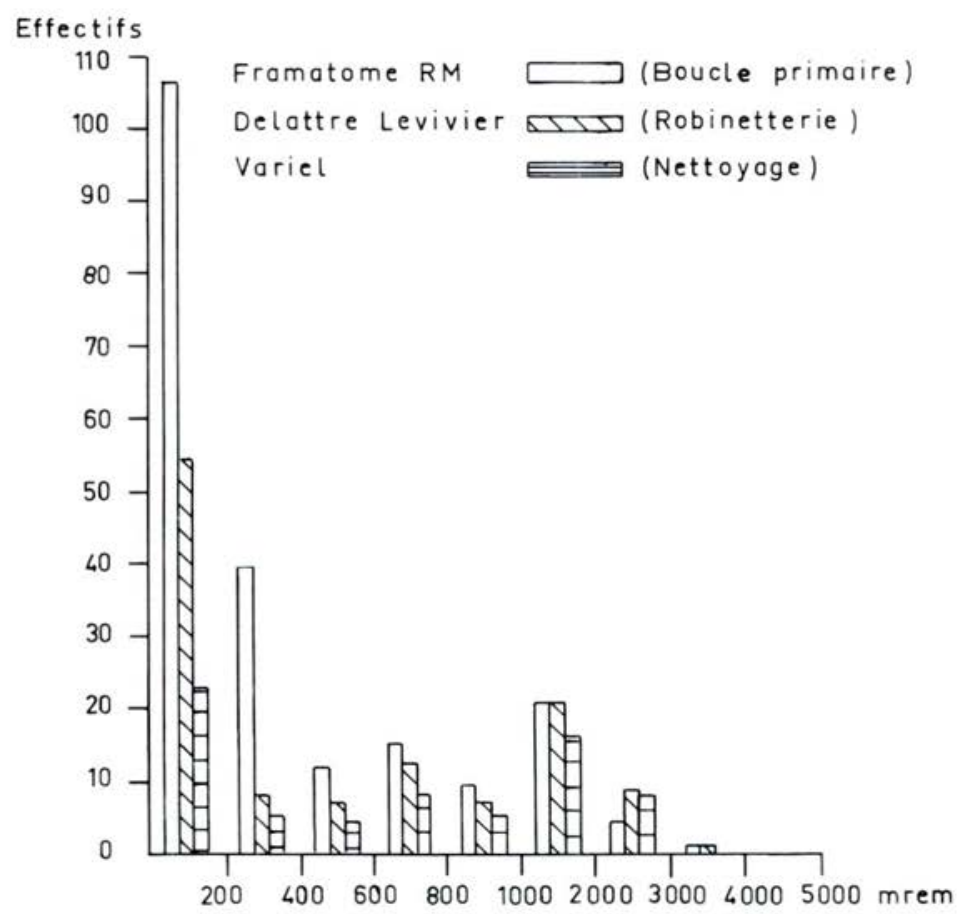

Fig. 7. - Répartition des doses/agent : ensemble des 4 révisions 1984 (3 entreprises).

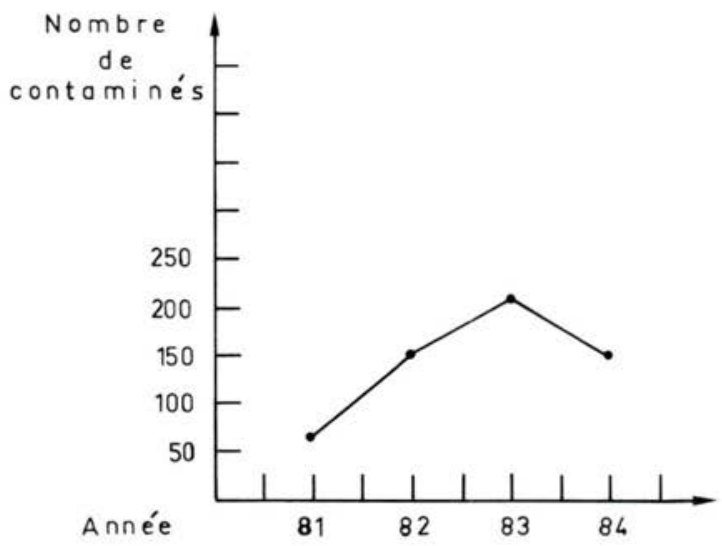

Fig. 8. - Evolution des contaminations corporelles. 
TABLEAU ।

Répartition des doses "entreprises" par chantier. Arrêt annuel, tranche 3, 1984.

\begin{tabular}{|l|c|}
\hline \multicolumn{1}{|c|}{ Observations } & $\begin{array}{c}\text { Doses cumulées } \\
\text { en rem }\end{array}$ \\
\hline Réacteur & \\
- Ouverture, fermeture & 11 \\
- Manutention combustible & 1 \\
- Changement broches, tubes guides & 25 \\
- Divers réacteur & 13,5 \\
Générateurs de vapeur & \\
- Ouverture, fermeture trous d'homme & 1 \\
- Pose, dépose des tapes d'obturation & 10,5 \\
- Inspection des tubes & 3,5 \\
- Divers GV & 10 \\
Circuit primaire & \\
- Pompes primaires & 1,5 \\
- Robinetterie & 6,5 \\
- Divers & 2,5 \\
Circuits RRA, RCV et divers & \\
- Robinetterie RRA & 0,5 \\
- Robinetterie RCV & 5,5 \\
- Autres robinetteries & 8 \\
- Entretien divers & 5 \\
- Inspections (hors tubes GV) & 5 \\
- Nervitudes, échafaudages & 4,5 \\
- Poseyages dépose calorifuges & 7,5 \\
& \\
\hline
\end{tabular}

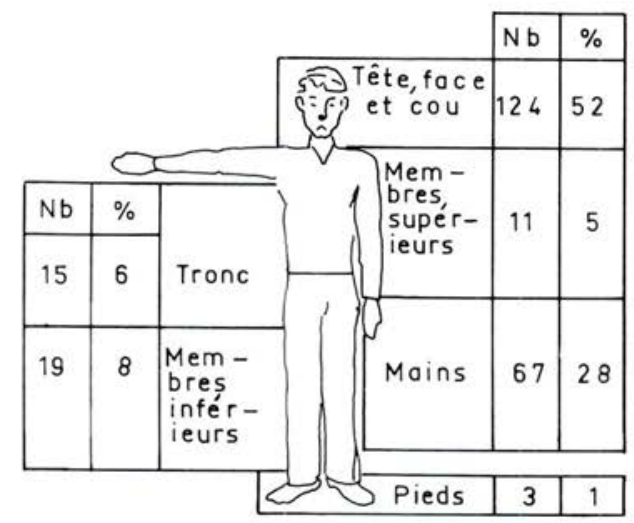

Fig. 9. - Nombre, siège et pourcentage des contaminations reçues par le personnel d'entreprises pour l'année 1984.

NB : 1 cas de contamination corporelle, relevé dans la figure 8 , peut donner lieu dans cette figure à plusieurs sièges de contamination (par ex. : tête + mains). 


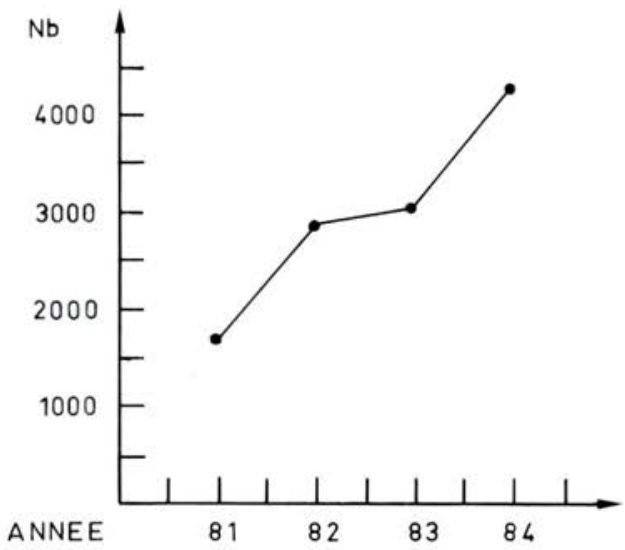

Fig. 10. - Surveillance radiologique des entreprises. Nombre des anthropogammamètries.

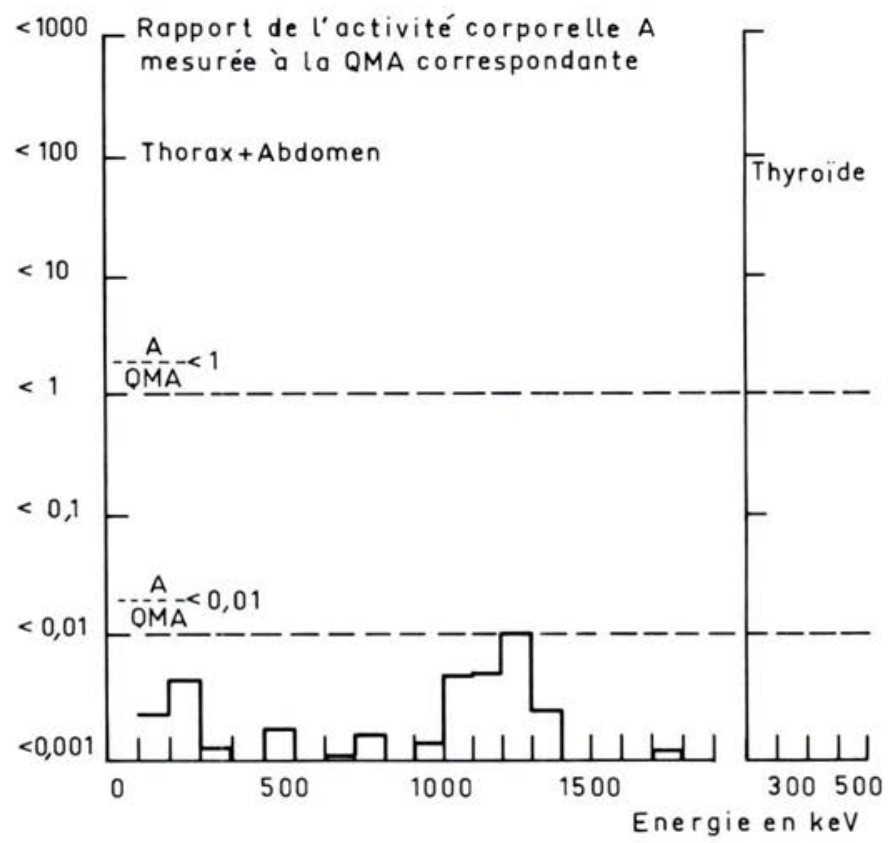

Le médecin de la centrale devra prendre contact avec le SCPRI.

Fig. 11. - Examen anthropogammamétrique réglementaire de dépistage. 